\title{
ORIGINAL
}

\section{CALIDAD DE VIDA RELACIONADA CON LA SALUD Y OBESIDAD EN UN CENTRO DE ATENCIÓN PRIMARIA}

\author{
Miguel Angel Barajas Gutiérrez (1), Elisa Robledo Martín (1), Nuria Tomás García (1), Teresa \\ Sanz Cuesta (2), Pilar García Martín (1) e Inmaculada Cerrada Somolinos (1). \\ (1) Centro de Salud Dr. Mendiguchía Carriche. Leganés. Madrid. \\ (2) Unidad de Investigación. Dirección de Atención Primaria. IX Área Sanitaria. Leganés (Madrid).
}

\section{RESUMEN}

Fundamento: Evaluar la asociación entre la obesidad y dos aspectos de la calidad de vida relacionada con la salud: autopercepción de salud y bienestar psicológico en pacientes adscritos a un centro de salud.

Métodos: Estudio transversal descriptivo sobre 167 pacientes entre 20 y 70 años, con índice de masa corporal $\geq 30$. Se realizó entrevista clínica para la obtención de las variables sociodemográficas y clínicas de cada paciente y la administración de 2 cuestionarios: 1) Perfil de Salud de Nottingham (PSN) y 2) Psychological General Well-Being (PGWB) índex.

Resultados: Las puntuaciones medias del PSN indicaron un deterioro global moderado de la salud percibida en las pacientes obesas siendo las áreas afectadas «dolor», «movilidad física» $y$ «reacciones emocionales». Las variables asociadas a una peor puntuación fueron el sexo femenino, la edad superior a 50 años, el bajo nivel de estudios y la presencia de patología psiquiátrica o artrosis. La puntuación total media del PGWB indica un deterioro pequeño del bienestar psicológico. El sexo femenino, la ansiedad y el bajo nivel de estudios se asociaron, asimismo, a peores puntuaciones.

Conclusiones: En nuestra población, se identifica un perfil de pacientes obesos con mala calidad de vida imujeres de edad superior a 50 años con bajo nivel de estudios y presencia de patología psiquiátrica o artrosis) sobre los que se plantea la conveniencia de realizar una actuación prioritaria.

Palabras clave: Obesidad. Calidad de vida. Encuesta de salud. Enfermedades crónicas. Salud percibida. Bienestar psicológico.

\section{ABSTRACT \\ Quality of Life Related to Health and Obesity at a Primary Care Center}

Background: To evaluate the association between obesity and two aspects of health-related quality of life: self-perception of health status and psychological well-being in patients assigned to a primary care center.

Methods: 167 patients between 20 and 70 ycars old with a body mass index $\geq$ were included in the study. A clinical interview was carried out to get sociodemographic and clinical data and to administer two questionnaires: 1) Nottingham Health Profile (NHP) and 2) Psychological General Well-being (PGWB) índex.

Results: The mean scores of the NHP indicated a moderate overall impairment of perceived health in females. Pain, emotional reactions and physical mobility were the affected dimensions. Females, age group over 50, low level of education and the presence of psychiatric pathology or osteoarthritis were significantly associated with worse scores. The mean scores of PGWB indicates a small impairment of psychological wellbeing. In this case, worse scores were associated with females, anxiety and low lcvel of cducation.

Conclusions: In the study population, we identify a profile of obese patients with worse quality of life: women with age over 50, low level of education and the presence of psychiatric pathology or osteoarthritis. We think it would be a good idea to achieve a prior performance towards them.

Key words: Obesity. Quality of life. Perceived health. Psychological well-being.
Correspondencia:

Miguel Angel Barajas Gutiérrez

Centro de Salud Dr. Mendiguchía Carriche.

Plaza de la Comunidad de Madrid. 3

28912 Leganés (Madrid)

Teléfono: 6880933

Fax: 6887545

\section{INTRODUCCIÓN}

La obesidad es la enfermedad metabólica más prevalente en los países industrializados. Su importancia radica tanto en la morbilidad que se le asocia como en la mortalidad que conlleva'. Su tratamiento es difícil 
con una tasa de recaídas que en algunas series alcanza el $90 \%{ }^{2}$.

Hasta la pasada década, la investigación en el campo de la obesidad estuvo enfocada casi exclusivamente sobre dos aspectos: por un lado, la asociación entre sobrepeso y morbimortalidad y, por otro, el tratamiento de la misma ${ }^{3}$. No obstante, en los últimos años y al igual que ha sucedido con otros trastornos crónicos ha despertado gran interés el estudio de la calidad de vida relacionada con la salud (CVRS) o salud percibida ${ }^{4}$ como un complemento importante para la valoración integral del paciente obeso. Existen estudios ${ }^{3.5}$ que demuestran que la CVRS del paciente obeso se deteriora en varios aspectos: capacidad física, bienestar psicológico, funcionamiento social, etc.

Para la valoración de la CVRS se utilizan básicamente dos tipos de cuestionarios: genéricos (permiten su aplicación en diferentes patologías) y específicos (diseñados para un trastorno concreto) ${ }^{6}$. Ambos tipos de instrumentos han sido desarrollados mayoritariamente en EE.UU, y en el Reino Unido. No obstante, en los últimos años, algunos de ellos han sido adaptados del inglés al castellano y posteriormente validados ${ }^{7-11}$. Aunque la CVRS del paciente obeso ha sido ampliamente estudiada fuera de nuestro país ${ }^{12-14}$, en nuestro medio son muy escasos trabajos similares $^{15}$.

El objetivo del presente estudio es, en primer lugar, evaluar la asociación entre obesidad y dos aspectos de la CVRS: la autopercepción general de salud y el bienestar psicológico mediante escalas de medida en pacientes adscritos a un centro de atención primaria y, en segundo lugar, intentar identificar las variables que se asocian con un deterioro de aquella.

\section{SUJETOS Y MÉTODO}

Se diseñó un estudio descriptivo transversal sobre una muestra obtenida aleatoria- mente entre todos los obesos registrados en el centro de salud Doctor Mendiguchía Carriche de Leganés (Madrid) en junio de 1996. Previamente a la selección de la muestra se llevó a cabo una exhaustiva revisión de las historias de usuarios mayores de 14 años, con el fin de detectar pacientes que, aún cumpliendo los criterios diagnósticos de obesidad -índice de masa corporal (IMC) $\geq 30-$, no estuvieran registrados. El cálculo del tamaño muestral se realizó para estimar la media de la puntuación total del PGWB índex, con una precisión de 3 puntos y un nivel de confianza del $95 \%$. Se asumió una desviación típica de 18 observada en la revisión bibliográfica ${ }^{7}$. El tamaño estimado fué de 177 sujetos. Esta muestra se duplicó previendo unas pérdidas importantes por varios motivos: tratarse de un estudio realizado sobre población adscrita a un centro de salud, el tipo de patología a estudiar y por posibles errores en la base de datos. No pudo determinarse antes del muestreo qué pacientes no cumplían los criterios de inclusión al no estar registrados en la base de datos todas las patologías que podían causar su exclusión del estudio. Por tanto, una vez obtenida la muestra y tras revisión de las historias clínicas, se seleccionó definitivamente a los pacientes que cumplían los siguientes criterios de inclusión: IMC $\geq 30$, edad entre $20 \mathrm{y}$ 70 años y ausencia de cualquier otro trastorno crónico, con la única excepción de aquellos que, por estar relacionados íntimamente con la propia obesidad (diabetes, HTA, artrosis), podrían afectar a la calidad de vida del paciente obeso, así como trastornos emocionales aparecidos posteriormente al comienzo de la obesidad, debido a la posibilidad de que éstos estuvieran relacionados con la misma.

Entre julio y diciembre de 1996 los pacientes incluidos fueron contactados telefónicamente o, cuando ésto no fué posible, mediante una carta personalizada, siéndoles explicados los motivos del estudio y requiriendo su colaboración. Los pacientes que aceptaron participar fueron citados en el centro para una entrevista personal, realizada 
por un médico residente de $3^{\text {er }}$ año de $\mathrm{Me}$ dicina Familiar y Comunitaria, en la que se recogieron, en primer lugar, las siguientes variables: sexo, edad, peso y talla (para el cálculo del IMC actualizado), edad de comienzo de la obesidad, situación de convivencia, nivel socioeconómico ${ }^{16}$ valorado en dos apartados: nivel de estudios y situación laboral (renunciándose a recoger la información sobre el nivel de ingresos por la dificultad de obtener datos fiables), intentos previos de adelgazar, ingesta nocturna, patologías concomitantes relacionadas con la obesidad (HTA, diabetes y artrosis) e historia psicológica (presencia de ansiedad y/o depresión posteriores al comienzo de la obesidad e ingesta de psicofármacos en el mes previo a la realización del estudio). Posteriormente les fueron administrados los siguientes cuestionarios: 1) la versión española del Perfil de Salud de Nottingham (PSN) ${ }^{9}$ que consta de 38 ítems y es un instrumento de medida genérico que refleja el nivel de sufrimiento en seis dimensiones del estado de salud (energía, dolor, reacciones emocionales, sueño, aislamiento social y movilidad física). Las puntuaciones de cada dimensión son los porcentajes de respuestas afirmativas siendo el recorrido de las mismas de 0 (ningún sufrimiento) a 100 (máximo nivel de sufrimiento), para cada dimensión; 2) la versión española del Psychological General Well-Being (PGWB) índex ${ }^{7}$, que es un instrumento que refleja los sentimientos de bienestar o de enfermedad. Consta de 22 ítems agrupados en seis dimensiones (ansiedad, depresión, estado de ánimo positivo, vitalidad, autocontrol, salud general). Cada ítem puntúa de 1 a 6 (de menor a mayor bienestar) por lo que el recorrido de la puntuación global oscila de 22 (máximo malestar) a 132 (máximo bienestar), aunque para la mejor comprensión de los resultados aquella puede trasladarse a una escala 0-100. Las puntuaciones parciales van de 3 a 18 cuando la dimensión consta de 3 ítems (depresión, autocontrol y salud general), de 4 a 20 si son 4 (estado de ánimo positivo y vitalidad) y de 5 a 30 en la dimensión que consta de 5 ítems (ansiedad).
Ambos cuestionarios fueron autoadministrados salvo en los pacientes que no sabían leer o tenían dificultades visuales.

A los pacientes que no aceptaron participar en el estudio se les preguntó el motivo de su negativa. En este grupo se analizaron las variables conocidas (cdad y sexo) que fueron comparadas con las de los pacientes incluidos en el estudio.

\section{Análisis estadístico}

Se utilizó el programa SSPS para Windows para el análisis de los datos. Se realizó un análisis descriptivo de las variables sociodemográficas y clínicas mediante la media y la desviación estándar así como con el cálculo de proporciones. Para la comparación de los resultados del PSN se han utilizado los valores obtenidos en la población general de Barcelona de edad superior a 40 años ${ }^{9}$. En el caso del PGWB índex no ha sido posible encontrar una población de referencia en nuestro entorno por lo que se ha utilizado población sana sueca encontrada en la revisión bibliográfica ${ }^{17}$. La comparación se ha realizado de forma separada por sexos ya que se sabe que en ambos cuestionarios hay diferencias significativas entre varones y mujeres $^{18}$. Se ha calculado el tamaño del efecto de la asociación entre la obesidad y las puntuaciones de los cuestionarios como el cociente de la diferencia entre la media de la población obesa y sana dividido por el promedio de las desviaciones estándar de ambas poblaciones ${ }^{14}$, considerándose un efecto pequeño, moderado y grande a valores superiores a $0,20,0,50$ y 0,80 respectivamente ${ }^{19}$. Para el análisis comparativo de las puntuaciones medias del PSN se utilizaron pruebas no paramétricas (Wilcoxon-MannWhitney o Kruskal-Wallis), dada la ausencia de normalidad. En cuanto al $P G W B$ index se utilizaron pruebas paramétricas ( $\mathrm{t}$ de Student o análisis de varianza) o no parámetricas en cada caso, según la presencia o no de normalidad. Para la comparación de variables cuantitativas con las puntuaciones de los 
cuestionarios se aplicó el coeficiente de correlación de Spearman. El nivel de significación utilizado ha sido 0.05 .

\section{RESULTADOS}

De los 12.500 usuarios adultos que en junio de 1996 constaban en el registro del centro, 835 eran obesos. De la muestra de 350 que se obtuvo inicialmente, 52 pacientes $(15 \%)$ no cumplían los criterios de inclusión. No se pudo contactar con 54 pacientes (15\%). Del resto, $63(18 \%)$ no aceptaron participar -alegando el $38 \%$ de ellos motivos laborales-y otros 14 (4\%) fueron excluidos por haber dejado de cumplir el criterio de peso. La edad media de los que no participaron en el estudio fué $50 \pm 13$ años. Entre ellos, había 70 varones (38\%) y 113 mujeres (62\%). En ambas variables se encontraron diferencias estadísticamente significativas entre los sujetos incluidos y no incluidos, siendo estos últimos más jóvenes $(\mathrm{p}<0,001)$ y con mayor proporción de varones $(p<0.0007)$. Al final del estudio cumplimentaron los cuestionarios $167 \mathrm{pa}$ cientes cuyas características sociodemográficas y clínicas se exponen en la tabla 1.

La edad media fué de $54 \pm 10$ años. El $60 \%$ de los pacientes $(\mathrm{n}=98)$ presentaba una obesidad leve (IMC < 35) y el $9 \%(n=15)$ tenía un IMC > 40 (obesidad mórbida). Con independencia del estado civil, menos del 5\% de los pacientes vivían solos. El 84\% de los sujetos estudiados tenía un nivel de estudios primario o inferior. Respecto a la situación laboral, se observaron diferencias importantes entre los dos sexos. Así, el 77\% de las pacientes obesas eran amas de casa y sólo el $17 \%$ de las mismas realizaba trabajo remunerado. Por el contrario, el $58 \%$ de los varones tenía trabajo, refiriendo un $8 \%$ encontrarse en situación de desempleo. La ingesta nocturna se daba en menos del $5 \%$ de los casos.

\section{Tabla 1}

Características sociodemográficas y clínicas de la población de estudio

\begin{tabular}{|c|c|}
\hline & $N(\%)$ \\
\hline \multicolumn{2}{|l|}{ Edad } \\
\hline$\leq 40$ & $18(10,8)$ \\
\hline $41-50$ & $34(20,4)$ \\
\hline $51-60$ & $60(35,9)$ \\
\hline$\geq 61$ & $55(32,9)$ \\
\hline \multicolumn{2}{|l|}{ Sexo } \\
\hline Varón & $36(21,6)$ \\
\hline Mujer & $131(78,4)$ \\
\hline IMC & $34,82 \pm 4,02 *$ \\
\hline Edad de comienzo & $29,9 \pm 13,8^{*}$ \\
\hline \multicolumn{2}{|l|}{ Convivencia } \\
\hline Vive solo & $8(4,2)$ \\
\hline Convive & $159(95,2)$ \\
\hline \multicolumn{2}{|l|}{ Nivel de estudios } \\
\hline Sin estudios & $42(25,1)$ \\
\hline Primarios & $98(58,7)$ \\
\hline Secundarios & $22(13,2)$ \\
\hline Bachillerato & $4(2,4)$ \\
\hline Universitarios & $1(0,6)$ \\
\hline \multicolumn{2}{|l|}{ Situación laboral } \\
\hline Empleado & $44(26,3)$ \\
\hline Jubilado & $16(9,6)$ \\
\hline Desempleado & $5(3)$ \\
\hline Ama de casa & $101(60,5)$ \\
\hline \multicolumn{2}{|l|}{ Ingesta noctuma } \\
\hline sí & $7(4,2)$ \\
\hline no & $160(95,2)$ \\
\hline \multicolumn{2}{|l|}{ Intentos previos de adelgazar } \\
\hline sí & $110(65,9)$ \\
\hline no & $57(34,1)$ \\
\hline \multicolumn{2}{|l|}{ Historia psicológica } \\
\hline Ansiedad (sí / no) & $28(17) / 139(83)$ \\
\hline Depresión (sí / no) & $18(11) / 149(89)$ \\
\hline Ingesta de psicofármacos (sí/no) & $33(20) / 134(80)$ \\
\hline \multicolumn{2}{|l|}{ Patologías concomitantes } \\
\hline HTA (sí / no) & $82(49) / 85(51)$ \\
\hline Artrosis (sí / no) & $59(35) / 108(65)$ \\
\hline Diabetes (sí / no) & $20(12) / 147(88)$ \\
\hline
\end{tabular}

* media \pm desviación estándar; n: tamaño muestral. 
En las tablas 2 y 3 se muestran las puntuaciones medias y desviaciones estándar de las distintas dimensiones de los cuestionarios PSN y PGWB índex respectivamente, por sexos, tanto en la población obesa como en las poblaciones de referencia y el tamaño del efecto calculado. Las puntuaciones medias del PSN reflejan una asociación entre la presencia de obesidad y un deterioro de la salud percibida distinta en los dos sexos. Así, mientras que las mujeres obesas puntúan significativamente peor que la población de referencia en 3 áreas: «dolor», «movilidad física» y «reacciones emocionales», en el caso de los varones sólo se encuentran diferencias en el área «movilidad física». La puntuación media global del PGWB índex indica un efecto pequeño de la obesidad sobre el bienestar psicológico en los dos sexos. Las dos dimensiones más alteradas en este caso fueron «depresión» y «salud general», más llamativamente en varones.
La relación entre las puntuaciones del PSN y PGWB índex y el grado de obesidad queda reflejado en la tabla 4 . En el caso del PSN, al separar los valores de IMC por rangos $(<35$, entre 35 y 40 y $>40$ ) no se encontraron diferencias significativas entre los mismos, salvo en la dimensión «movilidad física» y la puntuación total. Cabe señalar, no obstante, la importante diferencia encontrada en las dimensiones «dolor»y «energía» a pesar de no superar el umbral de la significación estadística. En cuanto al PGWB índex, sólo la «vitalidad» aparece relacionada negativamente con el grado de obesidad.

El análisis bivariante entre el resto de variables y la salud percibida señala que las mujeres, los pacientes mayores de 50 años, los que tenían patología psiquiátrica o artrosis y los sujetos con bajo nivel de estudios presentaron, en varias dimensiones, puntuaciones del PSN significativamente peores que los va-

Tabla 2

Puntuaciones medias y desviaciones estándar de las escalas de medida de pacientes varones obesos y población masculina de referencia y tamaño del efecto

\begin{tabular}{|c|c|c|c|}
\hline & \multicolumn{2}{|c|}{ Media $(D E)$} & \multirow[b]{2}{*}{ Tamaño del efecto } \\
\hline & Pacientes obesos $(n=131)$ & Población general* & \\
\hline \multicolumn{4}{|l|}{ Perfil de Salud de Nottingham } \\
\hline Energía & $11,1(26,4)$ & $8,5(21,6)$ & 0,10 \\
\hline Dolor & $9,3(14,4)$ & $9,1(20,8)$ & 0,01 \\
\hline Reacciones emocionales & $16.3(23,3)$ & $16,1(19,5)$ & 0,00 \\
\hline Sueño & $10(21,6)$ & $17,6(25,4)$ & $-0,32$ \\
\hline Aislamiento social & $6,6(16,5)$ & $5,7(13,0)$ & 0,06 \\
\hline Movilidad física & $16,3(20,6)$ & $10,6(17,4)$ & 0,30 \\
\hline \multicolumn{4}{|l|}{ PGWB index } \\
\hline Ansiedad & $26,8(4,3)$ & $23,8(4,5)$ & 0,66 \\
\hline Depresión & $13,1(0,7)$ & $16,5(2,2)$ & $-2,31$ \\
\hline Estado de ánimo positivo & $17,3(3,5)$ & $17,4(3,7)$ & $-0,01$ \\
\hline Autocontrol & $15,5(3,0)$ & $15,9(2,2)$ & $-0,14$ \\
\hline Salud general & $13(1,7)$ & $16,7(1,7)$ & $-1,92$ \\
\hline Vitalidad & $20,4(3,7)$ & $18,6(2,8)$ & 0,52 \\
\hline TOTAL & $106,3(13,2)$ & $109,2(13,7)$ & -0.21 \\
\hline
\end{tabular}

* Para el PSN n=5799 y para PGWB índex $n=6417$. DE: desviación estándar 
Tabla 3

Puntuaciones medias y desviaciones estándar de las escalas de medida en pacientes obesas y población femenina de referencia y tamaño del efecto

\begin{tabular}{|c|c|c|c|}
\hline & \multicolumn{2}{|c|}{ Media $(D E)$} & \multirow[b]{2}{*}{ Tamaño del efecto } \\
\hline & Pacientes obesos $(n=131)$ & Población general * & \\
\hline \multicolumn{4}{|l|}{ Perfil de Salud de Nottingham } \\
\hline Energía & $20,8(31,3)$ & $19,0(31,9)$ & 0,11 \\
\hline Dolor & $36.1(31,1)$ & $21,5(28,4)$ & 0,49 \\
\hline Reacciones emocionales & $32,9(23,0)$ & $25,0(24,6)$ & 0,66 \\
\hline Sueño & $25(28,4)$ & $28,2(31,4)$ & 0,10 \\
\hline Aislamiento social & $8,3(16,0)$ & $8.6(16,5)$ & $-0,03$ \\
\hline Movilidad física & $28,4(22,3)$ & $18,8(21,9)$ & 0,43 \\
\hline \multicolumn{4}{|l|}{ PGWB índex } \\
\hline Ansiedad & $22,9(4,8)$ & $23,1(4,5)$ & 0,03 \\
\hline Depresión & $13,1(1,3)$ & $15,5(2,8)$ & $-1,16$ \\
\hline Estado de ánimo positivo & $14.6(3,8)$ & $15,4(3,9)$ & $-0,20$ \\
\hline Autocontrol & $14,8(2,7)$ & $14,6(3,3)$ & 0,06 \\
\hline Salud general & $13,3(2,0)$ & $15,0(3,5)$ & $-0,60$ \\
\hline Vitalidad & $17,1(4,2)$ & $15,6(4,3)$ & 0,34 \\
\hline TOTAL & $96,1(13,8)$ & $99,5(19,2)$ & $-0,20$ \\
\hline
\end{tabular}

* Para el PSN n=5799 y para PGWB índex n=6417

DE: desviación estándar.

Tabla 4

Puntuaciones medias de las escalas de medida del cstado de salud según el grado de obesidad

\begin{tabular}{|c|c|c|c|}
\hline & \multicolumn{3}{|c|}{$I M C$} \\
\hline & $<35(n=98)$ & $35-40 \quad(n=54)$ & $>40(n=15)$ \\
\hline \multicolumn{4}{|l|}{ PSN } \\
\hline Energía & 26,4 & 34,4 & 41,6 \\
\hline Dolor & 14.6 & 21,6 & 35,5 \\
\hline Reacciones emocionales & 28,1 & 29 & 39,2 \\
\hline Sueño & 20,8 & 20,7 & 32 \\
\hline Aislamiento social & 6,1 & 9,2 & 16 \\
\hline Movilidad física & 22 & 30 & $35^{\mathrm{a}}$ \\
\hline TOTAL & 21 & 25,5 & $34,8^{\mathrm{a}}$ \\
\hline \multicolumn{4}{|l|}{ PGWB índex } \\
\hline Ansiedad & 23,8 & 24 & 22,8 \\
\hline Depresión & 13,1 & 13,1 & 13 \\
\hline Estado de ánimo positivo & 15,5 & 15 & 14,3 \\
\hline Autocontrol & 15 & 14,8 & 14,8 \\
\hline Salud General & 13,2 & 13,2 & 13,8 \\
\hline Vitalidad & 18,4 & 17 & $16,2^{\mathrm{a}}$ \\
\hline TOTAL & 99,2 & 97,5 & 95,2 \\
\hline
\end{tabular}

" $\mathrm{p}<0.05$. 
rones, los menores de 50 años, los que no presentaban comorbilidad y los que tenían un nivel alto de estudios (tabla 5). También se observaron diferencias significativas en algunas dimensiones en función de la situación laboral (peor las amas de casa y los desempleados), la ingesta nocturna y el haber realizado intentos previos de adelgazar (peor ante la presencia de ambas situaciones). No se encontraron diferencias significativas o fueron de escasa entidad en las puntuaciones del PSN en función de la presencia de HTA, diabetes, la edad de comienzo de la obesidad o el hecho de vivir en solitario.
En la tabla 6 se observa que en el sexo femenino, la existencia de patología psiquiátrica y la artrosis se asocian significativamente en el análisis bivariante a una peor puntuación del PGWB índex. No se encontraron diferencias, sin embargo, en función de la edad ni del resto de variables estudiadas con excepción de la situación laboral donde se aprecian diferencias significativas en la dimensión «vitalidad», puntuando más los trabajadores y jubilados respecto a desempleados y amas de casa $(\mathrm{p}<0.04)$.

Tabla 5

Puntuaciones medias del Perfil de Salud de Nottingham según sexo, edad, historia psicológica, artrosis y nivel de estudios

\begin{tabular}{|c|c|c|c|c|c|c|c|}
\hline & \multicolumn{7}{|c|}{ Dimensiones del PSN y puntuación total $(0-100)$} \\
\hline & $E$ & $D$ & $R E$ & $S$ & $A S$ & $M F$ & Total \\
\hline \multicolumn{8}{|l|}{ Sexo } \\
\hline Varones & $11,1^{\mathrm{a}}$ & $9.3^{\mathrm{c}}$ & $16,3^{\mathrm{c}}$ & $10^{\mathrm{b}}$ & 6,6 & $16,3^{\mathrm{b}}$ & $12,5^{\mathrm{c}}$ \\
\hline Mujeres & 20,8 & 36.1 & 32,9 & 25 & 8,3 & 28.4 & 26,8 \\
\hline \multicolumn{8}{|l|}{ Edad } \\
\hline$\leq 40$ & 12,9 & $9^{c}$ & $12,9^{\mathrm{b}}$ & 10 & 4,4 & $13,1^{\mathrm{c}}$ & $11,5^{\mathrm{c}}$ \\
\hline $41-50$ & 11,7 & 22,7 & 32 & 18,8 & 4,1 & 15,8 & 19,2 \\
\hline $51-60$ & 23,3 & 37,2 & 32,1 & 23,3 & 11,3 & 32,9 & 28,2 \\
\hline$\geq 61$ & 20 & 34,5 & 29 & 25,8 & 8 & 28,4 & 25,8 \\
\hline \multicolumn{8}{|l|}{ Ansiedad } \\
\hline Sí & 26,1 & $48,2^{b}$ & $48,8^{\circ}$ & $35^{3}$ & 9,2 & 32,1 & $35^{\mathrm{b}}$ \\
\hline No & 17,2 & 26,7 & 25,4 & 19,1 & 7,7 & 24,5 & 21,4 \\
\hline \multicolumn{8}{|l|}{ Depresión } \\
\hline Sí & 31,4 & $50^{\mathrm{b}}$ & $49,3^{\mathrm{c}}$ & $37,7^{\mathrm{b}}$ & 11,1 & $35,4^{\mathrm{a}}$ & $37,8^{\circ}$ \\
\hline No & 17,2 & 28 & 26,9 & 19,8 & 7,6 & 24,6 & 22 \\
\hline \multicolumn{8}{|c|}{ Psicofármacos } \\
\hline $\mathrm{Si}$ & 24,2 & $43,5^{\mathrm{a}}$ & 40,4 & $31,5^{\mathrm{a}}$ & 10,9 & $35,9^{\mathrm{b}}$ & $32,4^{\mathrm{b}}$ \\
\hline No & 17 & 27,3 & 26,8 & 19,6 & 7 & 22,9 & 21,6 \\
\hline \multicolumn{8}{|l|}{ Artrosis } \\
\hline Sí & $33,3^{\mathrm{c}}$ & $50^{\mathrm{c}}$ & $39,9^{\circ}$ & $33,2^{\mathrm{c}}$ & $12,5^{b}$ & $40^{c}$ & $36,3^{\mathrm{c}}$ \\
\hline No & 10,8 & 19.6 & 23,6 & 15,5 & 5,5 & 18 & 16.8 \\
\hline \multicolumn{8}{|c|}{ Nivel de estudios * } \\
\hline Bajo & $21,9^{b}$ & $33,2^{b}$ & $31,5^{\mathrm{b}}$ & 23,2 & $9,2^{\mathrm{a}}$ & $28,1^{\mathrm{b}}$ & $25,9^{\mathrm{c}}$ \\
\hline Alto & 2,4 & 15,7 & 18,5 & 14 & 1,4 & 13,8 & 12,6 \\
\hline
\end{tabular}

E: energía: D: dolor: RE: reacciones emocionales: S: sueño: AS: aislamiento social; MF: movilidad física; a: $p<0.05$; $b$ : $p<0.01$; c: $p<0.001$.

* Bajo: primarios o inferior; Alto: secundarios o superior. 
Tabla 6

Puntuaciones medias del PGWB índex según sexo, historia psicológica, artrosis y nivel de cstudios

\begin{tabular}{|c|c|c|c|c|c|c|c|}
\hline & \multicolumn{7}{|c|}{ Dimensiones del $P G W B$ índex y puntuación total } \\
\hline & $A N$ & $D$ & $E A$ & $A C$ & $S G$ & $V$ & Total \\
\hline \multicolumn{8}{|l|}{ Sexo } \\
\hline Varones & $26,8^{\mathrm{c}}$ & 13,1 & $17,3^{\mathrm{c}}$ & 15,5 & 13 & $20.4^{\circ}$ & $106,3^{\mathrm{i}}$ \\
\hline Mujeres & 22,9 & 13,1 & 14,6 & 14,8 & 13,3 & 17.1 & 96,1 \\
\hline \multicolumn{8}{|l|}{ Ansiedad } \\
\hline Sí & $200^{c}$ & 12,7 & $13,4^{b}$ & 14 & 13,5 & $14,9^{\mathrm{c}}$ & $88,7^{\mathrm{c}}$ \\
\hline No & 24,5 & 13,2 & 15,6 & 15,1 & 13,2 & 18,4 & 100,2 \\
\hline \multicolumn{8}{|l|}{ Depresión } \\
\hline Sí & $20,6^{b}$ & 13 & $13,1^{4}$ & $13,3^{\mathrm{a}}$ & 13,5 & $14,3^{c}$ & $88^{b}$ \\
\hline No & 24,2 & 13,1 & 15,4 & 15,2 & 13.2 & 18,2 & 99.5 \\
\hline \multicolumn{8}{|c|}{ Psicofármacos } \\
\hline Sí & $21^{\mathrm{c}}$ & 13.2 & $13,7^{\mathrm{a}}$ & 14.1 & 13,1 & $15.6^{\mathrm{c}}$ & $90,3^{c}$ \\
\hline No & 24,5 & 12,6 & 15.5 & 15.1 & 13,3 & 18,3 & 100,3 \\
\hline \multicolumn{8}{|l|}{ Artrosis } \\
\hline Sí & $22,3^{b}$ & 13,3 & $14,3^{\mathrm{i}}$ & 15 & 13,6 & $16,6^{\mathrm{a}}$ & 95,3 \\
\hline No & $2 \iota_{, 6}$ & 12,9 & 15,7 & 14,9 & 13,1 & 18,4 & 99,9 \\
\hline \multicolumn{8}{|c|}{ Nivel de estudios* } \\
\hline Bajo & 25,4 & $13,5^{\mathrm{a}}$ & 16 & 15,4 & 13 & 18,3 & 101,9 \\
\hline Alto & 23,5 & 13 & 15 & 14,9 & 13,3 & 17.7 & 97,6 \\
\hline
\end{tabular}

AN: ansiedad: D: depresión; EA: estado de ánimo positivo: AC; autocontrol; SG: salud general; V: vitalidad: a: $\mathrm{p}<0,05 ; \mathrm{b} ; \mathrm{p}<0,0 \mid$ : $\mathrm{e}: \mathrm{p}<0,001$.

* Bajo: primarios o inferior, Alto: secundarios o superior.

\section{DISCUSIÓN}

A diferencia de la mayoría de los trabajos publicados sobre valoración de la calidad de vida en el paciente obeso, tanto dentro como fuera de nuestro país, el presente estudio se ha realizado sobre población adscrita a un centro de atención primaria, lo que puede tener la ventaja, a nuestro parecer, de evitar el sesgo de selección que se podría producir al estudiar a pacientes que acuden a consultas de endocrinología o unidades de obesi$\mathrm{dad}^{12-15}$. Es interesante señalar, en este sentido, que el $34 \%$ de los pacientes estudiados nunca había realizado intentos de adelgazar, por lo que este trabajo supone un intento de evaluar la repercusión que tiene la obesidad sobre la calidad de vida de los obesos en general y no sobre un subgrupo de ellos. Cabe la posibilidad de que el hecho de consultar en un centro especializado esté rela- cionado con tener una peor autopercepción de salud o un menor bienestar psicológico.

Los resultados globales obtenidos demuestran que los pacientes obesos tienen asociado un deterioro moderado de la salud percibida respecto a la población general en el caso de las mujeres y mínimo en el de los varones. No obstante, dicho deterioro no afecta por igual a las distintas dimensiones. Así, mientras que «movilidad física» en ambos sexos y «dolor»y «reacciones emocionales», en el sexo femenino puntúan significativamente peor que en la población de referencia, no se ha encontrado diferencia alguna en «sueño»y «aislamiento social». Si se tiene en cuenta que a mayor edad se obtienen puntuaciones superiores ${ }^{18}$ y que la edad media de la población de referencia utilizada es superior a la de estudio, cabe suponer que en el presente trabajo existe una 
infraestimación de la asociación entre la obesidad y la salud percibida.

El hecho de que al desagregar las puntuaciones del PSN según el grado de obesidad, hallemos diferencias menores de las esperadas a la vista de los resultados globales, podría sugerir que el efecto negativo de la obesidad por sí misma sobre la calidad de vida es escaso. La estrecha relación encontrada entre la salud percibida y la presencia de patología psiquiátrica $\mathrm{y}$, sobre todo, la artrosis (patología íntimamente relacionada en su patogenia con la obesidad), señalaría a éstas como las máximas responsables del deterioro de la salud percibida del paciente obeso. No obstante, la pequeña asociación encontrada entre el grado de obesidad y el deterioro de la salud percibida puede ser debido a que el estudio carezca de potencia para verificar diferencias importantes puesto que, por una parte, en el grupo con IMC $\geq 40$ sólo hay 15 pacientes $y$, por otra, no se han incluido pacientes con sobrepeso (IMC entre 25 y 30 ) lo que probablemente habría aumentado la rentabilidad del trabajo.

No resulta fácil comparar los resultados de salud percibida obtenidos con los de otros estudios publicados debido al uso, en estos últimos ${ }^{12-15}$, de diferentes escalas de medida. A pesar de ello y puesto que los distintos cuestionarios están divididos en dimensiones no muy diferentes, se aprecia en todos los estudios una tendencia general a presentar al dolor y la movilidad física como las áreas de la salud percibida más deterioradas en el paciente obeso. Los resultados de nuestro estudio corroboran dicha tendencia. La fuerte asociación entre grado de obesidad y disminución de la autopercepción de salud puesta de manifiesto en otros trabajos ${ }^{13.15}$, quizá no sea tan evidente en el nuestro. En ese sentido, el PSN se ha mostrado como un instrumento relativamente poco sensible para detectar el deterioro de la salud percibida en personas «poco enfermas» ${ }^{3}$.
En relación a otros estudios realizados en nuestro país en los que se ha utilizado el PSN como instrumento para la valoración de la asociación entre salud percibida y otros trastornos crónicos y tcniendo presente la limitación que supone la sobrerrepresentación del sexo femenino en la muestra de obesos, llama la atención que en las dimensiones «dolor», «reacciones emocionales» $\mathrm{y}$ «movilidad física», las personas obesas estudiadas presentaron puntuaciones similares o superiores en algunos casos a pacientes con cardiopatía isquémica estable y enfermedad pulmonar obstructiva crónica lo que indicaría que, en términos de salud percibida, la obesidad (considerada, en muchas ocasiones, una enfermedad de escasa importancia) se podría equiparar a enfermedades de reconocido carácter discapacitante ${ }^{20,21}$.

Si bien la puntuación global del PGWB índex supera el valor 95 (límite inferior para ser considerada como bienestar) ${ }^{22}$, los valores obtenidos en ambos sexos son inferiores a los hallados en población sana ${ }^{17}$.

Los valores del PGWB índex según los valores del IMC por rangos son congrucntes con los globales en el sentido de no hallar diferencias significativas, con excepción de la «vitalidad» y con un bajo nivel de significación. Todo ello apuntaría (al igual que sucede con la salud percibida) a que la obesidad, de forma aislada, tiene una repercusión escasa sobre el bienestar psicológico. El sexo femenino, la ansiedad y poseer un nivel de estudios bajo son los factores que más favorecerían el malestar psicológico. Para la generalización de los resultados hay que tener en cuenta las limitaciones que se han señalado anteriormente respecto a la no inclusión de personas con sobrepeso lo que probablemente habría aumentado la potencia del estudio.

No hay publicados estudios que valoren el bienestar psicológico en obesos mediante el PGWB índex. De la revisión de los trabajos que utilizan otras escalas de medida ${ }^{12-15}$ se desprende que existe cierta disparidad en los 
resultados que varían desde una importante afectación del bienestar psicológico hasta la casi total ausencia de deterioro. Nuestros resultados, con las salvedades anteriormente expuestas, están más cerca de estos últimos. Otros puntos diferenciales de nuestro estudio son la muy pequeña relación encontrada entre peor bienestar y grado de obesidad y la nula relación del bienestar con el tiempo de evolución del proceso ${ }^{13}$. En la comparación de los datos de nuestro estudio con otros publicados que utilizan el PGWB índex para valorar el bienestar psicológico en otras patologías crónicas, las puntuaciones de los pacientes obesos quedan a medio camino entre los trastornos que no alteran el bienestar psicológico (HTA) y los que lo hacen de forma notable (enfermedades gastrointestinales) $)^{23,24}$.

Dados los malos resultados a largo plazo que se consiguen habitualmente en el tratamiento del paciente obeso podría ser conveniente, como hacen otros autores ${ }^{14}$, intentar seleccionar de alguna forma a aquellos pacientes que por presentar peor calidad de vida pudieran poseer un mayor grado de motivación para realizar correctamente el tratamiento y obtener, en consecuencia, un beneficio superior.

\section{AGRADECIMIENTOS}

Agradecemos a los Dres. Jordi Alonso y Luis Prieto su autorización y consejos para el uso de la versión española del PSN, así como el habernos proporcionado los datos necesarios para efectuar la comparación con nuestra población.

Agradecemos al Dr. Xavier Badia su autorización para la utilización de la versión española del PGWB índex.

\section{BIBLIOGRAFÍA}

1. Sjöstrom LV. Morbidity of severely obese subjects. Am J Clin Nutr 1992; 55: 508-515.
2. Brownell KD, Marlatt GA, Lichtenstein E, Wilson GT. Understanding and preventing relapse. Am Psychol 1986; 41(7): 756-782.

3. Sullivan MBE, Sullivan LGM, Kral JG. Quality of life assessment in obesity: psysical, psychological, and social function. Gastroenterol Clin North Am 1987;16: 433-442.

4. Guyatt GH, Feeny DH, Patrick DL. Measuring health-related quality of life. Ann Intern Med 1993; 118: 622-629.

5. Wadden TA, Stunkard AJ. Social and psychological consequences of obesity. $\Lambda$ nn Intern Med $1985 ; 103: 1062-1067$.

6. Fitzpatrick R, Fletcher A, Gore S, Jones D, Spiegelhalter D, Cox D. Quality of life measures in health care. I: Applications and issues in assessment. BMJ 1992; 305: 1074-7.

7. Badia X, Gutiérrez F, Wiklund Y, Alonso J. Validity and reliability of the spanish version of the Psychological General Well-Being Index. Qual Life Res 1996; 5: 101-108.

8. Alonso J, Antó JM, Moreno C. Spanish version of the Nottingham Health Profile: translation and preliminary validity. Am J Public Health 1990; 80 : 704-708.

9. Alonso J, Prieto L, Antó JM. The Spanish version of the Nottingham Health Profile: a review of adaptation and instrument characteristics. Qual Life Res 1994; 3: 385-393.

10. Badia X, Alonso J. Adaptación de una medida de la disfunción relacionada con la enfermedad: la versión española del Sickness Impact Profile. Med Clin (Barc.) 1994; 102: 90-5.

11. Alonso J, Prieto L, Antó JM. La versión española del SF-36 Health Survey: un instrumento para la medida de los resultados clínicos. Med Clin (Barc.) 1995; 104: 771-776

12. Sullivan M, Karlsson J, Sjöström L, Backman L, Bengtsson, C, Bouchard C et al. Swedish obese subjects (SOS) - an intervention study of obesity. Baseline evaluation of health and psychosocial functioning in the first 1734 subjects examined. Int J Obes 1993; 17: 503-512.

13. Kolotkin RL, Head S, Hamilton M, Tse CK. Assessing impact of weight on quality of life. Obes Res $1995 ; 3: 49-56$.

14. Fontaine KR, Cheskin LJ, Barofsky I. Health-related quality of life in obese persons seeking treatment. J Fam Pract 1996; 43: 265-270. 
15. Formiguera X, Badia X, Aguilar G, Barbany M, Schiafino A, Cuatrecasas G et al. Quality of life and morbid obesity. Int J Obes 1996; 20 (suppl 4): 160 .

16. Alvarez Dardet C, Alonso J, Domingo A, Regidor E. La medición de la clase social en ciencias de la salud. Barcelona: SG-Editores; 1995.

17. Rose G, Bengtsson C. Effects of a health examination programme on quality of life and subjective well-being. Scand J Soc Med 1996; 2: 124131.

18. Ware JE, Keller SD. Interpreting general health measures. En: Spilker B, ed. Quality of life and pharmacoeconomics in clinical trials. Philadelphia: Lippincort-Raven Publishers, 1996 .p. 445460 .

19. Kazis LE, Anderson JJ, Meenan RF. Effects sizes for interpreting changes in health status. Med Care 1989; 27(3): S178-S189.

20. Alonso J, Antó JM, González M, Fiz JA, Izquierdo J, Morera J. Measurement of general health status of non-oxygen-dependent chronic obstruc- tive disease patients. Med Care 1992; 30: MS125MS135.

21. Permanyer-Miralda G, Alonso .J, Antó IM, Alijarde-Guimerá M, Soler-Soler J. Comparison of perceived health status and conventional functional evaluation in stable patients with coronary artery disease. J Clin Epidemiol 1991; 44: 779-786.

22. Dupuy HJ. The psychological general well-being (PGWB) index. In: Wenger NK, Mattson ME, Fuberg $\mathrm{CP}$, eds. Assessment of quality of life in clinical trials of cardiovascular therapies. New York: Le Jacq, 1994.

23. Walle PO, Westergren G, Dimenäs E, Olofsson B, Albrektsen T. Effects of $100 \mathrm{mg}$ of controlled-release metoprolol and $100 \mathrm{mg}$ of atenolol on blood pressure, central nervous system-related symptoms, and general well being. J Clin Pharmacol 1994; 34: 742-747.

24. Dimenäs $E$, Glise $H$, Hallerbäck, Hernqvist $H$, Svedlund, Wiklund I. Well-Being and gastrointestinal symptoms among patients referred to endoscopy owing to suspected duodenal ulcer. Scand J Gastroenterol 1995; 30: 1046-52. 